\title{
Identification Procedure for Model Parameter of Earth Pressure Balance System of Shield Using Genetic Algorithm
}

\author{
Zichang Shangguan \\ Institute of Marine and Civil Engineering \\ Dalian Ocean University \\ Dalian, China \\ shangguan@dlou.edu.cn
}

\author{
Shouju Li \\ State Key Laboratory of Structural Analysis for \\ Industrial Equipment \\ Dalian University of Technology \\ Dalian, China \\ lishouju@dlut.edu.cn
}

\begin{abstract}
The mathematical model of earth pressure balance system of shield machine is proposed that is based on the mass balance principal in chamber of shield and Hook law of soil material. The state equation of the system is used to depict the relationship between earth pressure in chamber of shield and angular velocity of screw conveyor. The differential form of mathematical model of earth pressure system is derived. In order to determine model parameters of soil pressure balance system of shield, the genetic algorithm is used and developed. Based on the measured data in laboratory experiments, the model parameters are estimated. The investigation shows that the predicted soil pressure in chamber of shield agree well with observed ones. The effectiveness of proposed parameter estimation procedure is validated by experimental investigations in laboratory.
\end{abstract}

Keywords-model parameter estimation; genetic algorithm; earth pressure balanced shield; experimental investigation; mathematical model

\section{INTRODUCTION}

The use of a shield machine has become the first choice when planning the excavation of a circular tunnel in urban environment. The basic advantages of shield machine are high safety and rapid excavation speed, with low overbreak (extra-excavation) and low manpower. Shield machine tunneling also has only a very small effect on the surrounding soil mass and constructions. Earth pressure balance shields provide continuous support of the tunnel face using freshly excavated soil, which completely fills up the work chamber under pressure. Shield machines have the advantages of limiting the disturbance to the surrounding ground and producing a smooth tunnel wall. This significantly reduces the cost of lining the tunnel, and makes them suitable to use in heavily urbanized areas. Urban tunnelling has the special requirement that the ground surface must be undisturbed. This means that ground subsidence must be avoided. The normal method of doing this in soft ground is to maintain the soil pressures during and after the tunnel construction. There is some difficulty in doing this, particularly in varied strata. The earth pressure balanced (EPB) shield gets its name because it is capable of holding up soft ground by maintaining a balance between earth and pressure. The shield operator and automated systems keep the rate of soil removal equal to the rate of machine advance. Thus, a stable environment is maintained. Yang proposed a thrust hydraulic system of shield tunneling machine with pressure and flow compound control scheme[1]. In the control system, the task of real-time measuring of the pressure and the displacement of hydraulic cylinder in each thrust group was accomplished by pressure sensor and displacement sensor respectively, forming the closed-loop pressure and speed control with proportional valves. Thrust system mathematical modeling including hydraulic system and multi-cylinder acting dynamics was presented[Yang et al (2007)]. Zhou developed a database of tunneling parameters and strata considering the shield tunneling practice of Guangzhou Rail Transit[2]. Based on the data a method of strata identification was studied by using neural network pattern recognition technology. Based on the analysis of the features of strata in shield tunneling and the data, a one-to-many mapping relation between strata and data was proposed[Zhou et al. (2009)]. Kim proposed an equation for the minimum support pressure as a function of friction angle, cohesion, lateral earth pressure coefficient, tunnel diameter, and tunnel depth[3]. The minimum support pressures are compared to the analytical solutions available from the literature[Kim et al. (2010)].Li proposed a generalized nonlinear time-varying dynamic model for the cutterhead driving system of a shield tunnel boring machine. A nonlinear multiple input and multiple output state-space dynamic model was also proposed. The dynamic model was simulated to analyze the driving system and the effects of system parameters such as gear backlash and transmission error, larger gear inertia, and load torque on the dynamic response of driving system were investigated as well[Li et al. (2010)] [4]. Acaroglu proposed a model to predict specific energy requirement of constant cross-section disc cutters in the rock cutting process by using fuzzy logic method. This model was based on experience and the database which consists of linear cutting test results that were generated over for many years at the Earth Mechanics Institute of the Colorado School of Mines[Acaroglu et al. (2008)][5]-[8]. 


\section{MODEL OF SOIL PRESSURE BALANCE SYSTEM IN SHIELD TUNNELING}

Earth Pressure Balance (EPB) tunneling machines excavate the soil with a rotating cutterhead, through which the soil passes into a pressurized head chamber. A screw conveyor extracts the soil from the head chamber, allowing control of the volume of soil excavated by the machine to balance the earth pressure in the head chamber. By this process the excavated soil in the pressurised head chamber supports the ground during excavation to provide stability and minimise ground movements.

The supporting pressure is achieved through control of the incoming and outgoing materials in the chamber, i.e., through regulation of the screw conveyor rotation and the excavation advance rate, as shown in Fig .1. The amount of earth removed by advancing the shield machine is expressed as follows

$$
V_{i}=A \int v d t=A v \Delta t
$$

Where $V_{\mathrm{i}}$ is amount of earth removed by advancing the shield machine, $v$ is the speed of shield machine tunneling, $A$ is the sectional area of the shield machine body. It is noted that the amount of earth removed by the shield machine is equal to the advanced volume of the machine. The amount of earth conveyed by the screw conveyor is expressed as follows

$$
V_{o}=\int q d t=q \Delta t
$$

Where $V_{\mathrm{o}}$ is the amount of conditioned soil conveyed by the screw conveyor. $q$ is the volumetric throughput of a screw conveyor.

$$
q=A_{s} \omega h \eta
$$

Where $h$ is screw pitch, $\omega$ is angular velocity of screw, $\eta$ is volumetric efficiency. The effective area of the screw conveyor is expressed as follows

$$
A_{s}=\pi\left(r_{s}^{2}-r_{f}^{2}\right)
$$

Where $r_{\mathrm{s}}$ is screw radius diameter, $r_{\mathrm{f}}$ is core or shaft radius.

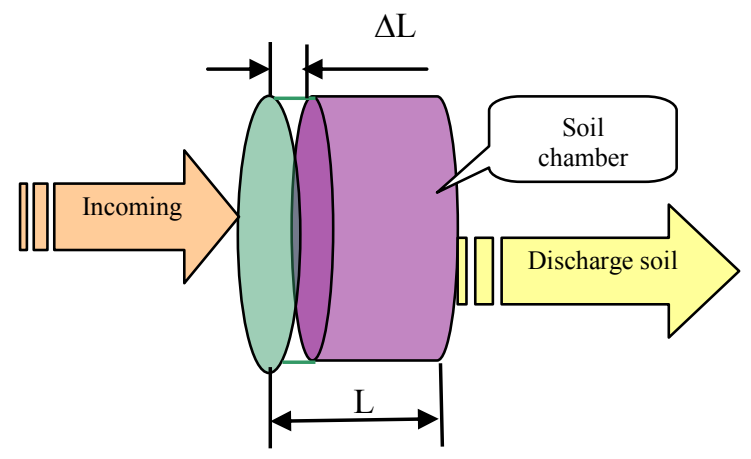

Figure 1. Computational model of soil strain in soil chamber

The amount of earth accumulating in the chamber is given by

$$
\Delta V=V_{i}-V_{o}
$$

The relationships between stress and strain, strain and amount of earth accumulating can be written as follows

$$
\begin{aligned}
& \Delta p=E_{t} \Delta \varepsilon \\
& \Delta \varepsilon=\frac{\Delta L}{L}=\frac{A \Delta L}{A L}=\frac{\Delta V}{V_{c}}
\end{aligned}
$$

Where $E$ is the tangent modulus of conditioned soil, $\Delta \mathrm{p}$ is the increment of earth pressure in the chamber. Combing (6) with (7) and (5), the relationship between the increment of earth pressure and the angular velocity of screw conveyor can be expressed as follows[Shangguan et al (2010)][9]

$$
\begin{aligned}
& \Delta p=\frac{E_{t}}{V_{c}}\left[A \int v d t-\int q d t\right] \\
& =\frac{E_{t}}{V_{c}}\left[A v-\frac{A_{s} \omega(t) h \eta}{2 \pi}\right] \Delta t
\end{aligned}
$$

(8) provides the foundation for controlling earth pressure of shield machine with optimization algorithm. The controlling problem of earth pressure of shield machine can be solved by optimization algorithm when the initial conditions and constraining conditions are known. The differential form of mathematical model of soil pressure system is expressed as follows

$$
p(t+\Delta t)=p(t)+\frac{E_{t}}{V_{c}}\left[A v-\frac{A_{s} \omega(t) h \eta}{2 \pi}\right] \Delta t
$$

When the dimensions of shield machine and soil mechanical properties are known the change of soil pressure in soil chamber versus rotating speed of screw conveyor can be computed.

\section{MODEL PARAMETER ESTIMATION PROCEDURES USING GENETIC ALGORITHM}

The solution of the inverse problem consists in obtaining a minimum of an objective function which is defined taking into account the mathematical structure of the material model and asset of experimental data. In order to determine some parameters of mechanistic model, the genetic algorithm and measuring data of system input and output are used. The objective function of parameter identification of mechanistic model is expressed as

$$
\min J=\sum_{k=1}^{n}\left[p_{m}(i)-p_{c}(i)\right]^{2}
$$

Where $J$ is objective function of parameter identification of mechanistic model, pm is earth pressure measured in bulkhead, $p_{c}$ is earth pressure calculated from mechanistic model, which is function of identified parameters. $n$ is data length for parameter identification. The unknown model parameters can be identified by using genetic algorithm.

A simple GA works on the chromosomes of the population and not on the decision variables. Individuals in 
the population are coded as strings, known as chromosomes, so that genotypes (chromosome values) are uniquely mapped on to the decision variables (known as phenotypes). There are many different schemes for chromosome encoding, such as binary coding, real-value coding and tree coding. Examination of a chromosome string in isolation usually yields no information about the problem to be solved. A chromosome needs to be decoded into its phenotypic values and the objective function (fitness) of the chromosome can then be evaluated. The search process in GAs operates on the encoding of the decision variables, rather than on the decision variables themselves, except in cases where the genotypes are identical with the phenotypes, as in the case of a real-value coding. It starts by generating chromosomes representing the initial population with a specific number of individuals. The fitness values of the individuals are evaluated on the basis of the objective function of the optimization problem. From here on, genetic evolution proceeds from generation to generation. In producing a new generation, the parents needed for breeding a new child are first selected according to their fitness values and put into the mating pool. The selection will ensure that high-fitted individuals have a high probability of being selected. Recombination takes place to produce children from the selected parents, often via some kind of crossover operations. The generated children can go through mutations, with some bits in the chromosome being mutated. The purpose of mutation, which is generally a background operator with a small probability, is to prevent premature convergence of the population. The GA sequence ends when either the maximum generations have been produced or other predefined termination conditions are satisfied. The average performance of individuals in the population is expected to increase during the evolution of the populations, as good individuals are preserved and bred with one another and the less-fit individuals die out[Yang et al. (2003)][10].

\section{EXPERIMENTAL INVESTIGATIONS}

The device is made of $1850 \mathrm{~mm}$ length tank with a 900 $\mathrm{mm}$ nominal inner diameter which is filled with soil. A $1000 \mathrm{~mm}$ long screw conveyor is coupled to the tank with an upward inclination of $30^{\circ}$ which is the average value usually used in EPB machines and the screw extends inside the tank to collect and discharge the soil. The diameter of the screw case is $160 \mathrm{~mm}$, the flights have a pitch of $128 \mathrm{~mm}$ and the screw shaft has a $50 \mathrm{~mm}$ diameter. The main dimensions and parameters of excavation chamber and screw conveyor are listed as Table 1 and 2 .

TABLE I. MAIN DIMENSIONS OF EXCAVATION CHAMBER

\begin{tabular}{ccccc}
\hline $\begin{array}{c}D( \\
\mathrm{m})\end{array}$ & $\begin{array}{c}L( \\
\mathrm{m})\end{array}$ & $\begin{array}{c}V(\mathrm{~mm} / \mathrm{m} \\
\text { in })\end{array}$ & $\begin{array}{c}A( \\
\left.\mathrm{m}^{2}\right)\end{array}$ & $\begin{array}{c}V_{c}( \\
\left.\mathrm{m}^{3}\right)\end{array}$ \\
\hline 0. & 0.1 & 20.0 & 0.6 & 0.08 \\
9 & 28 & & 36 & 14 \\
\hline
\end{tabular}

TABLE II. MAIN PARAMETERS OF SCREW CONVEYOR

\begin{tabular}{cccc}
\hline$r_{\mathrm{S}} / \mathrm{mm}$ & $r_{\mathrm{f}} / \mathrm{mm}$ & $h / \mathrm{mm}$ & $A_{\mathrm{S}} / \mathrm{mm}^{2}$ \\
\hline 160 & 50 & 128 & 18142.7 \\
\hline
\end{tabular}

Some experiments were performed in laboratory. During thrusting simulation, the thrusting speed is constant, $\mathrm{v}=20 \mathrm{~mm} / \mathrm{min}$. Fig .2 shows the variation of conveyor rotation speed versus time. The variation of chamber pressure versus time is measured, as shown in Fig .3. According to these measuring data, the model parameters of soil pressure balance system of shield machine are identified by using genetic algorithm, as shown in Table 3 .

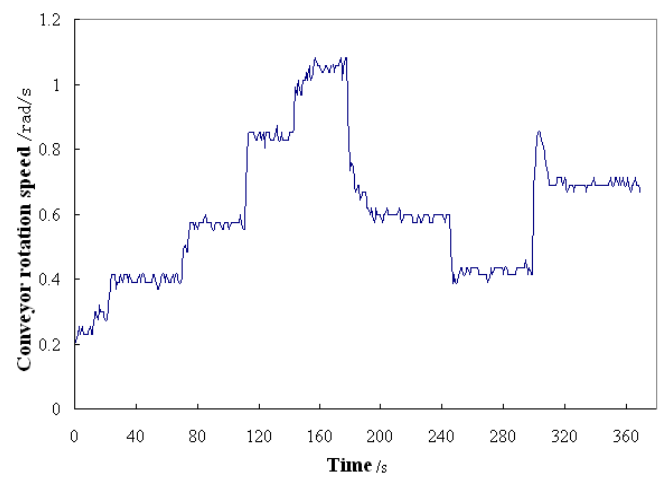

Figure 2. Variation of conveyor rotation speed versus time

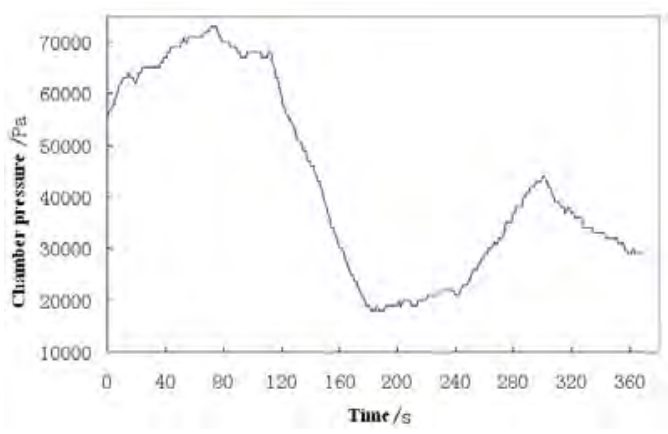

Figure 3. Variation of chamber pressure versus time

TABLE III. IDENTIFIED MODEL PARAMETERS

\begin{tabular}{ccc}
\hline Identified model parameters & $E_{\mathrm{t}} / k \mathrm{~Pa}$ & $\eta \%$ \\
\hline Identified values & 257.0 & 112 \\
\hline
\end{tabular}

Based on the identified model parameters, the variation of soil chamber pressure versus time is predicted. The comparison between forecast soil pressure and observed soil pressure is depicted in Fig .4. The investigation shows that the predicted soil pressure in chamber of shield agree well with observed ones. The effectiveness of proposed parameter inversion is validated by experimental investigations in laboratory.

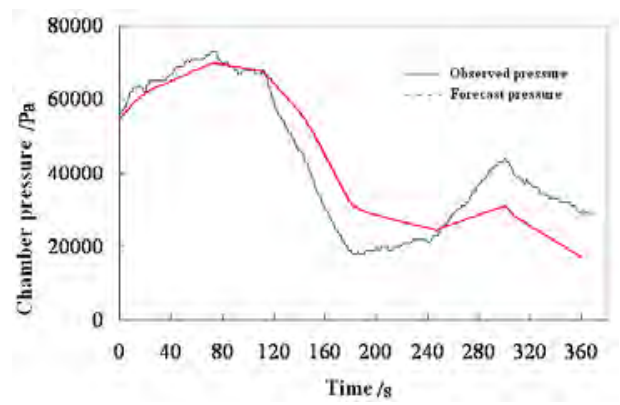

Figure 4. Comparison between forecast pressure and observed pressure 


\section{CONCLUSIONS}

A mathematical model for describing relationship between soil pressure in chamber of shield and angular velocity of screw conveyor is proposed. The genetic algorithm was used to estimate model parameters. The effectiveness of proposed parameter inversion is validated by experimental investigations in laboratory. The predicted soil pressure in chamber of shield agree well with observed ones, which indicates that the present inversion procedure has a good potential for model parameters estimation. There are several issues remaining to be addressed in the future research. The proposed parameter estimation procedure should be applied to the identification of model parameters using field experiment data. In order to increase computation efficiency, some hybrid inversion procedures should be developed

\section{ACKNOWLEDGMENT}

This research is supported by the National Key Basic Research and Development Program of China (No. 2013CB035402) and the Fundamental Research Funds for the Central Universiti es (Granted Num: DUT13LK14).

\section{REFERENCES}

[1] Huayong Yang, Hu Shi, Guofang Gong, et al. (2009) Electrohydraulic proportional control of thrust system for shield tunneling machine, Automation in Construction 18(7), pp.950-956.
[2] Xiwen Zhou, Yimin Xia, Jing Xue (2009) Neural Network Strata Identification Based on Tunneling Parameters of Shield Machine, Lecture Notes in Computer Science 5928, pp.392-401.

[3] S. H. Kim, F. Tonon (2010) Face stability and required support pressure for TBM driven tunnels with ideal face membraneDrained case, Tunnelling and Underground Space Technology 25(5), pp. 526-542.

[4] XianHong Li, HaiBin Yu, MingZhe Yuan(2010) Dynamic Modeling and Analysis of Shield TBM Cutterhead Driving System, Journal of Dynamic Systems, Measurement, and Control 132(4): 044504, pp.1-14.

[5] O. Acaroglu, L. Ozdemir, B. Asbury(2008) A fuzzy logic model to predict specific energy requirement for TBM performance prediction, Tunnelling and Underground Space Technology 23(5), pp. 600-608.

[6] M. A. Fazal, M. Imaizumi, S. Ishida(2005) Estimating groundwater recharge using the SMAR conceptual model calibrated by genetic algorithm, Journal of Hydrology 303(1-4), pp.56-78.

[7] J. Yen, J. C. Liao, B. Lee(1998) A hybrid approach to modeling metabolic systems using a genetic algorithm and simplex method, IEEE Systems, Man, and Cybernetics Society, 28(2), pp.173-191.

[8] M. Martin, M. J. Wilmut, N. R. Chapman (1999) A hybrid simplex genetic algorithm for estimating geoacoustic parameters using matched-field inversion, IEEE Journal of Oceanic Engineering 24(3), pp.358-369.

[9] Zichang Shangguan, Shouju Li, Maotian Luan(2010) Model Reference Control for Bulkhead Pressure of EPB Shield in Tunneling, Journal of Convergence Information Technology 5(1), pp.111-115.

[10] Y. Y. Yang, D. A. Linkens, M. Mahfouf(2003) Genetic algorithms and hybrid neural network modelling for aluminium stress-strain prediction, Journal of Systems and Control Engineering 217(7), pp.7-22. 